\title{
Virtual Fires \\ Experiences with Developing a LBGK Based \\ Real Time Tunnel Fire Simulator for Virtual Environments
}

\author{
Wilhelm Brandstätter and Christian Redl \\ Christian-Doppler-Laboratory for Applied Computational Thermofluiddynamics, \\ Mining University Leoben, \\ Franz-Josef-Strasse 18, 8700 Leoben, Austria
}

\begin{abstract}
In the VIRTUAL FIRES project a LBGK based simulator is coupled to a virtual reality environment for a interactive real time simulation of tunnel fires. The simulator not only has to deal with turbulent buoyant flows but it must also be able to calculate a big number of timesteps each second for the real time visualisation. The general concept as well as some implementation details are highlighted. Furthermore first results fire simulation in a real scale tunnel containing two trucks are presented.
\end{abstract}

\section{Introduction}

Recent fire accidents in tunnels (Mont Blanc tunnel, Tauern tunnel) [1] have raised attention on fire prevention and fire fighting means. Real fire tests in tunnels are very costly and are environmentally not friendly due to the production of toxic smoke. Therefore they can only be carried out occasionally. In addition fire fighters are endangered if they exercise with real fires.

All these aspects leaded to the idea of a virtual tunnel fire. Therefore a simulator is developed and connected to a virtual environment which allows a realistic visualisation of smoke and flame spread.

In this paper the authors want to describe their experiences in developing an interactive real time tunnel fire simulator for virtual environments based on a Lattice BGK model [2]. The reasons for selection a Lattice Boltzmann based model for simulation a large scale problem are explained. Furthermore first results obtained with the simulation program are presented. The VIRTUAL FIRES simulator is an example how to successfully apply the Lattice BGK method to real world large scale problems. Detailed information about the project and the consortium can be found at the projects web page [3].

\section{General System Layout}

The VIRTUAL FIRES simulator consists of three main parts :

P.M.A. Sloot et al. (Eds.): ICCS 2003, LNCS 2657, pp. 1062-1071, 2003.

(c) Springer-Verlag Berlin Heidelberg 2003 
- A database contains all information about the tunnel (geometry, ventilation system, ...) and data concerning the fire representation. Additionally the results of the simulation are stored in the database.

- A Lattice BGK based simulator performs the calculation of turbulent temperature and smoke spread.

- The results are displayed in a virtual reality (VR) environment (CAVE, HMD) using algorithms for a realistic visualisation of smoke.

The VIRTUAL FIRES user is able to retrieve all information from the database from within the VR environment. A rough model of the tunnel is displayed in the visualisation device and the user can place an arbitrary number of vehicles in the computational domain. For each object which should be set on fire information about energy and smoke release are obtained from the database.

During the simulation the results are sent to the visualisation engine upon request. Ideally this would be 25 times per second. The user is able switch on and off the ventilation device of the tunnel interactively from within the VR environment and observe the impact on the smoke spread.

The results used for visualisation are usually not stored due to memory constrains. However, the results are stored frequently in the database allowing the user to restart the simulation at certain points in time using different ventilation settings.

\section{Numerical Model}

\subsection{Reason for Selecting a Lattice BGK Model}

Although there are several drawbacks of the LBGK model there are also some strong reasons to select it.

- The regular grid structure and explicit nature allows a very efficient parallelisation. The parallel version scales almost linearly up to a large number of processors.

- The grid generation can be done automatically within the simulator. This is especially important as the potential users do not have much experience in grid generation.

- The regular structure of the computational grid is similar to the grid used for visualisation. The interpolation process from the grid used in the simulation and the one used for visualisation is straightforward.

- As a large number of simulation results are required each second for a real time visualisation explicit methods are suitable.

The authors want to stress that the decision for using a Lattice Boltzmann method instead of a more conventional finite volume formulation is also based on the innovative aspect of applying a LGBK model to large scale problems. 


\subsection{Turbulent Lattice BGK Model}

Currently two implementations of the Lattice BGK model are under investigation for the VIRTUAL FIRES project.

The first one, which is described in this paper, is a standard incompressible formulation of the Lattice Boltzmann equation in combination with thermally induced body forces.

The second LBGK model used in the VIRTUAL FIRES project is based on the low Mach number formulation for variable density flows of Filippova and Hänel [4] extended to account for buoyancy effects (as done for laminar jet diffusion flames by Lee et al [5]).

For both models turbulence effects are taken into account by using a Smagorinsky type sub grid scale model including turbulence production due to buoyancy [6] [7]. The spread of smoke can additionally be modelled using an additional distribution function for the smoke concentration.

In this paper only the incompressible formulation is decribed as investigations using the variable density model are in progress.

The incompressible Lattice Boltzmann equation can be written as :

$$
p_{i}\left(t+1, \mathbf{x}+\mathbf{e}_{i}\right)=p_{i}(t, \mathbf{x})-\frac{1}{\tau_{\nu}}\left(p_{i}(t, \mathbf{x})-p_{i}^{e q}(t, \mathbf{x})\right)
$$

where the equilibrium distributions are given by

$$
p_{i}{ }^{e q}=t_{i}\left\{p+p_{0}\left(\frac{\mathbf{e}_{i \alpha} \mathbf{u}_{\alpha}}{c_{s}{ }^{2}}+\frac{\mathbf{u}_{\alpha} \mathbf{u}_{\beta}}{2 c_{s}{ }^{2}}\left(\frac{\mathbf{e}_{i \alpha} \mathbf{e}_{i \beta}}{c_{s}{ }^{2}}-\delta_{\alpha \beta}\right)\right)\right\}+S_{i}
$$

The energy equation is treated as a scalar transport equation for the temperature within the LBGK framework:

$$
g_{i}\left(t+1, \mathbf{x}+\mathbf{e}_{i}\right)=g_{i}(t, \mathbf{x})-\frac{1}{\tau_{D}}\left(g_{i}(t, \mathbf{x})-g_{i}^{e q}(t, \mathbf{x})\right)+S_{T, i}
$$

with the equilibrium distributions given by

$$
g_{i}{ }^{e q}=t_{i} T\left\{1+\left(\frac{\mathbf{e}_{i \alpha} \mathbf{u}_{\alpha}}{c_{s}{ }^{2}}+\frac{\mathbf{u}_{\alpha} \mathbf{u}_{\beta}}{2 c_{s}{ }^{2}}\left(\frac{\mathbf{e}_{i \alpha} \mathbf{e}_{i \beta}}{c_{s}{ }^{2}}-\delta_{\alpha \beta}\right)\right)\right\}
$$

In the above equations $p_{0}$ is a reference pressure, $T$ is the temperature, $p_{i}$ and $g_{i}$ are the distribution functions, $\mathbf{e}_{i}$ are the lattice vectors and $S_{i}$ and $S_{T, i}$ are the corresponding source terms. The source term for the transport equation for the temperature is of course only added within the predefined fire region. The lattice dependent weighting factors for the D3Q19 model are $t_{0}=1 / 3$ for the rest particle distribution, $t_{1-6}=1 / 18$ for the cartesian directions and $t_{7-18}=1 / 36$ for the diagonal directed lattice vectors. The lattice speed of sound is $c_{s}=1 / \sqrt{3}$.

The hydrodynamice quantities can be obtained by taking the moments of the particle distribution functions :

$$
\rho=\sum_{i} p_{i}
$$




$$
\begin{aligned}
\rho \mathbf{u} & =\sum_{i} p_{i} \mathbf{e}_{i} \\
T & =\sum_{i} g_{i}
\end{aligned}
$$

The turbulent relaxation parameter $\tau_{\nu}$ and $\tau_{D}$ are calculated as follows :

$$
\begin{aligned}
\tau_{\nu} & =\frac{6\left(\nu+\nu_{t}\right)+1}{2} \\
\tau_{D} & =\frac{6\left(D+D_{t}\right)+1}{2}
\end{aligned}
$$

The turbulent transport coefficients are calculated by using a Smagorinsky sub grid scale model [7]:

$$
\begin{gathered}
\nu_{t}=C_{s} \Delta \sqrt{\left(2 \overline{S_{i j}} \bar{S}_{i j}-\frac{\beta}{P r_{t}} \frac{\partial T}{\partial x_{j}} g_{k} \delta_{k j}\right)} \\
D_{t}=\frac{\nu_{t}}{P r_{t}}
\end{gathered}
$$

In the above equations $C_{s}$ is the Smagorinsky constant, $\Delta$ is the filter width, which is equal to the lattice spacing and $P r_{t}$ is presumed turbulent Prandtl number. $\beta$ is the volumetric expansion coefficient and $g_{k}$ is the vector of gravity.

Since the density differences away from the fire source are relatively small the Boussinesq approximation is assumed to be valid. The resulting source term $S=-\beta g\left(T-T_{r e f}\right)$ is added to the equilibrium distribution functions (see. eq. (2)).

A comparison between the results obtained with both LBGK models and a detailed decription of the variable density implementation are presented in a forthcoming paper [8].

\subsection{Representation of the Fire}

For the fire modelling a volumetric heat source model is used [9]. A fire source region is predefined and source terms of smoke and energy are added to the evolution equations within this region. As for many engineering applications the details of the combustion process are not important for tunnel fires. Usually the combustible material is not known and the reactions are far too complex to be considered in detail. For the design of tunnel ventilation systems empirical smoke and energy release curves are used. These curves are available for a number of scenarios (e. g. including different types of vehicles like cars or HGVs) and show the smoke and heat release over time. A typical curve [10] used for the VIRTUAL FIRES project is shown in fig 1.

Radiation heat transfer is included using the radiative fraction approach described in [11]. In this approach the thermal radiation in the fluid is ignored and a fixed fraction (typically between 0.2 and 0.4 ) of the total heat release rate is assumed to be lost without affecting the temperature field. 


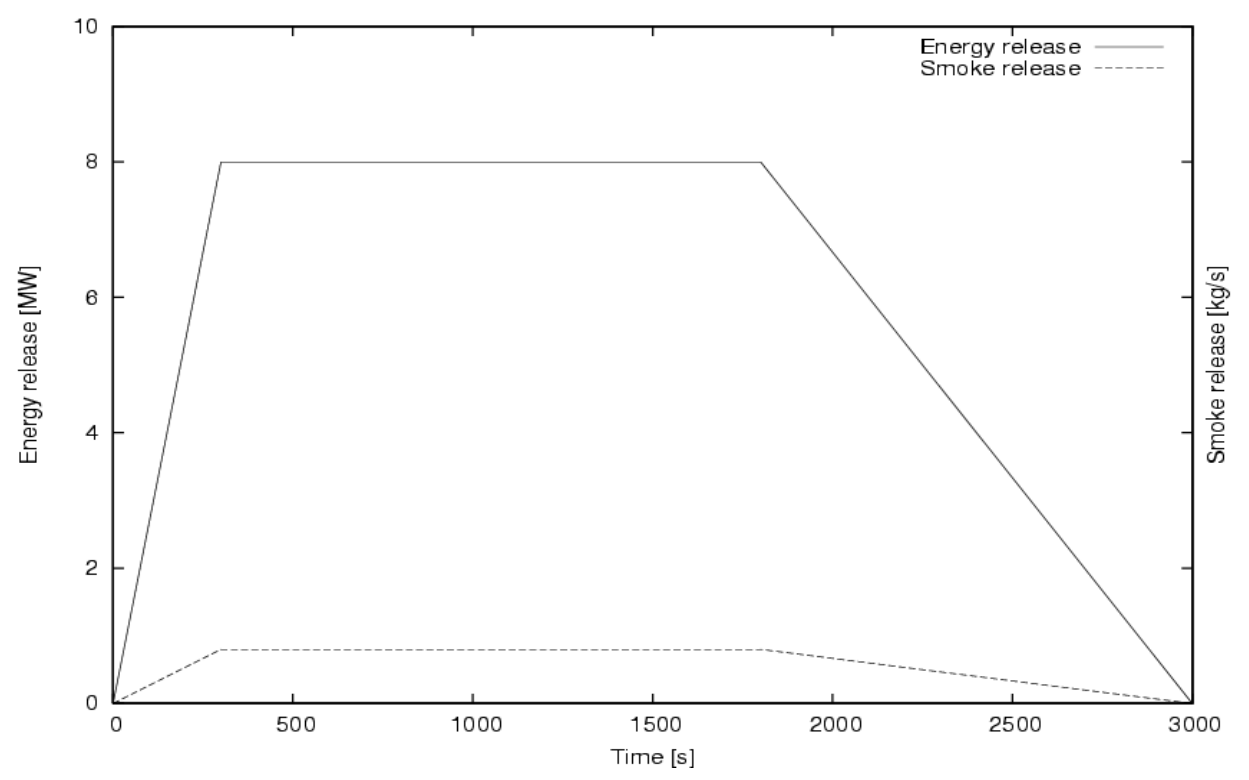

Fig. 1. Energy and smoke release curve for a small heavy goods vehicle [10]

\subsection{Multidimensional Coupling}

To extend the computational domain the 3D model is coupled to a one dimensional two zonal model on both sides. The two zonal model represents the flow field in the tunnel as one upper hot zone below the ceiling and one lower cold zone near the floor [12]. The coupling between the 3D and 1D model along the interface follows the idea described in [13] and is done as follows : The three dimensional cross section is subdivided into two areas. The hydrodynamic variables are averaged across both areas using an averaging procedure which accentuates the spatial centre. These averaged values are used as boundary conditions for the $1 \mathrm{D}$ sequence which on the other hand provides the boundary values for the 3D section. The extension of the areas are dynamically adjusted during the simulation. The one-dimensional transport equations are solved by an simple finite difference scheme. Fig. 2 shows an outline of the multi dimensional coupling and the domain decomposition for parallel computing.

\section{Parallelisation and Communication}

\subsection{Parallelisation}

The parallelisation can be done very efficiently using the MPI library. To achieve the maximum performance the computational domain is currently restriced to 
a rectangular shape. The domain decomposition is done either using a onedimensional coordinate decomposition or a multi-dimensional coordinate bisection method. Prior to the domain decomposition the expected surface/volume ratios for both methods are roughly estimated and the simulator automatically selects the algorithm which is best suited for the available number of processors.

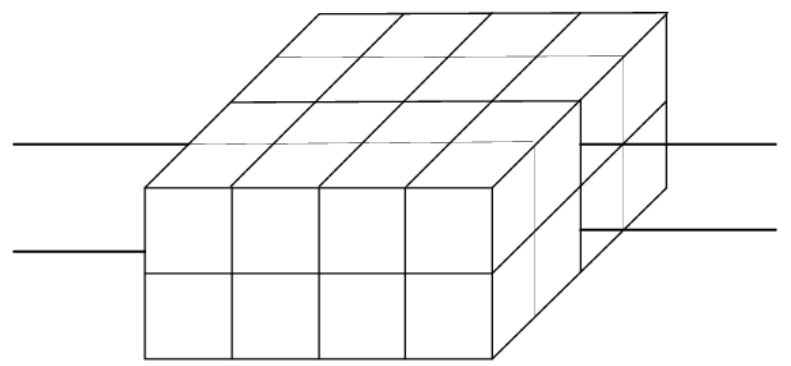

Fig. 2. Example of domain decomposition in combination with multi dimensional coupling

\subsection{Communication}

The communication between virtual environment and the simulation program is done via Unix sockets using the communication libraries provided by the visualisation program [14]. For output of the results three options were considered:

- Each processor writes its data to a file belonging to the processor. The data are subsequently gathered within the visualisation part.

- Each processor writes its data to one common output file.

- One processor gathers all data from all other processors and writes it to one output file.

It was decided to use the third option where only the master processor is concerned with the data transfer. It allows a better separation of the simulation and visualisation part and not every processor needs to have access to a writeable file system mounted. Furthermore it is possible to run the simulation on a remote host.

\subsection{Performance}

The simulation program aims at delivering results of 25 time steps per second for the visualisation. Due to the explicit nature of the Lattice Boltzmann method the computational time step may be smaller than the one needed for visualisation. Preliminary simulations show that currently about 50000 cells can be calculated in real time using the available computing facilities at the Parallel Data Center 
at KTH Stockholm [15]. The authors found this parallel performance remarkable as due to the real time requirements the computational domains for each processor are rather small. Additionally the gathering of results on the processor responsible for the $\mathrm{I} / \mathrm{O}$ can take as long as ten times the calculation of one time step.

\section{Results}

\subsection{Testcase A: Tunnelfire}

As an examplary testcase the tunnelfire experiment by Xue et al [9] is presented.

The experimental set up is as follows :

The tunnel section is $6[\mathrm{~m}]$ long with a rectangular cross section of $0.3[\mathrm{~m}]$ height and $0.9[\mathrm{~m}]$ width. The fire source of $3.15[\mathrm{~kW}]$ is located $1.5[\mathrm{~m}]$ from the inlet and occupies an volume of $0.18 \times 0.15 \times 0.01[\mathrm{~m} 3]$. The ventilation velocity is about $0.13[\mathrm{~m} / \mathrm{s}]$, ambient air temperature is $300[\mathrm{~K}]$. In contrast to the numerical study in the original paper the heat loss to the tunnel walls is neglected and the wall temperature is set to $300[\mathrm{~K}]$. The temperature distributions are reported at distances $0.9[\mathrm{~m}], 3.3[\mathrm{~m}]$ and $5.1[\mathrm{~m}]$ from the inlet.

Xue et al [9] pointed out that none of the combustion model used in their study are able to predict velocity and temperature fields in all test cases equally well.

Due to the limitations of the volumetric heat source model it is not expected to achive very good quantitative agreement with the experimental data. Nevertheless the general flow patterns are resolved well by the Lattice BGK model: Despite the longitudinal ventilation there is some backflow against the ventilation direction. A strong stratification of hot gases beneath the tunnel ceiling occurs. The time averaged temperature profiles obtained by the Virtual Fires simulator are in reasonable agreement with the ones obtained by Xue et al [9] using finite volume method in combination with a volumetric heat source model. Nevertheless both methods perform relatively poor in predicting the measured temperatures at locations near the fire. This is mainly due to the crude representation of the fire in the volumetric heat source model. An interesting observation is that the Boussinesq approximation performs surprisingly well for this testcase. As the main point of interest of the Virtual Fires project is to study the spreading of hot gases and not the combustion process itself it is justified to use this approximation at least in the first stage. As mentioned before detailed results will be published elsewhere [8].

\subsection{Testcase B: Burning Truck within a Tunnel}

The results of a typical simulation run using the VIRTUAL FIRES simulator are presented below.

The configuration shown in fig. 3 consists of a section of a tunnel containing two exhaust air outlets located at the ceiling. This corresponds to a usual installation in a modern full cross ventilated tunnel [16]. A certain underpressure is 
assigned to the extraction openings in order to model the removal of hot gases. The effects of natural ventilation are taken into account by applying a pressure difference along the tunnel axis. In reality this longitudinal velocity is induced by differences in altitude of the two tunnel portals or wind outside the tunnel.

Two heavy goods vehicles (HGV) are placed within the tunnel. One trailer is defined as fire zone and set on fire. The smoke and energy release curve shown in fig. 1 is used.

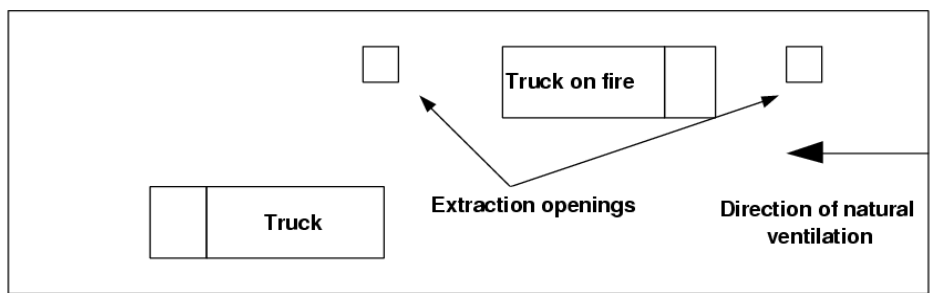

Fig. 3. Outline of the simulated scenario

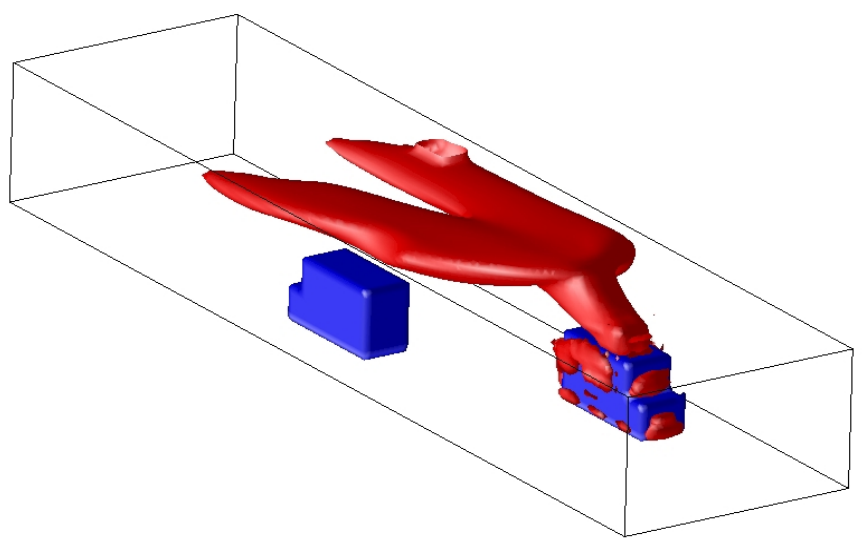

Fig. 4. Ventilation system acting in normal mode. Temperature $=423[\mathrm{~K}]$

Fig. 4 - 5 depict the results of two simulated scenarios in a relatively early stage after the fire onset. All figures show instantaneous temperture isosurfaces. The two scenarios are as follows: 


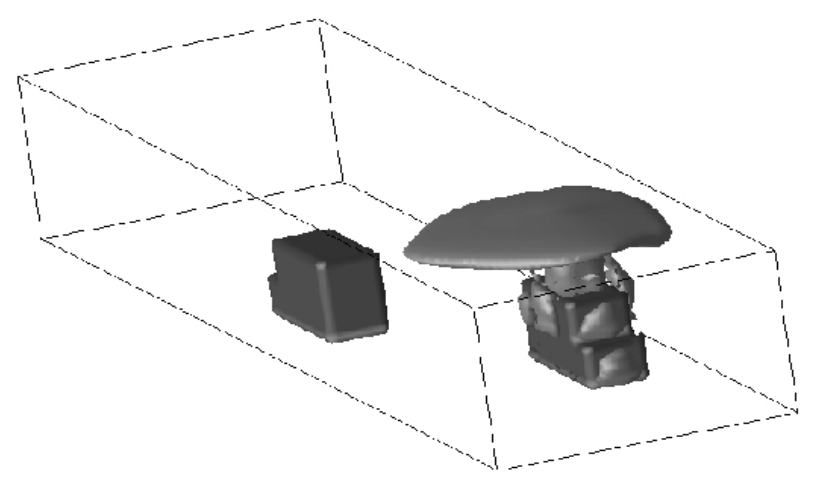

Fig. 5. Ventilation system acting in emergency mode. Temperature $=423[\mathrm{~K}]$

In the first one the ventilation system is acting in normal mode and natural ventilation is acting. As expected the hot gases are driven in direction of the natural ventilation. The extraction opening located in front of the burning truck has minor influence on the spread of smoke and the one located behind the truck is able to extract only a small fraction of hot gases. In fig. 4 the 423 [K] surface is depicted.

In the second scenario represents the emergency mode. The ventilation system is working at full extraction capacity. As can be seen from fig. 5 a big fraction of hot gases are extracted by the ventilation opening in front of the burning truck. The zone of hot gases is very narrow compared to the first scenario.

The computational domain contains about 300000 cells. Despite the rough resolution the results are in reasonable agreement compared to results obtained using a commercial CFD program with standard $k-\epsilon$-model [17].

\section{Conclusion}

The selection of a LBGK method working on an uniform grid enables the simulation of complex thermo-fluiddynamic problems such as tunnel fires. The presented results are very promising and the authors hope that the VIRTUAL FIRES simulatior will assist fire fighters and tunnel operator in dealing with fire hazards in tunnels. Future developments will focus on the improvement of interaction between virtual environment and simulation. It is planned to include the possibility of using fire nozzles which can be steered by the user interactively. 


\section{Acknowledgements.}

The authors want to express their graditude to the European Commission for financing the EU project VIRTUAL FIRES (IST-2000-29266) and to all project partners.

\section{References}

1. Hörhan, R. : Tunnel Accidents and their Impact on Relevant Guidlines in Austria. In: Pischinger, R.(ed.): Proceedings Int. Conf. Tunnel Safety and Ventilation. VKM-THD Mitteilungen Vol. 80. Verlag der Technischen Universität Graz, $\operatorname{Graz}(2002), 1-8$.

2. Chen, S., Doolen, G. D. : Lattice Boltzmann Method for Fluid Flows. Annu. Rev. Fluid Mech. 30 (1998), 329-364.

3. http://www.virtualfires.org

4. Filipova, O., Hänel, D. : A Novel Lattice BGK Approach for Low Mach Number Combustion. J. Comp. Phys. 158 (2000), 139-160.

5. Lee, T., Lin, C.-L., Chen, L.-D. : Lattice Boltzmann Simulation of Laminar Jet Diffusion Flame. Proceedings of the 2002 Spring Technical Meeting, Central Sates Section/The Combustion Institute, Knoxville, Tennessee, April 7-9, 2002.

6. Orlandi, P. : Fluid Flow Phenomena. Moreau, R. (ed.) : Fluid Mechanics and its Applications, Kluwer (2000), pp 356.

7. Peng, S.-H., Davidson, L. : Comparison of Sub-Grid-Scale Models in LES for Turbulent Convection Flow with Heat Transfer. Proceedings of 2. EF Conference in Turbulent Heat Transfer, Manchester (UK), May 31 - June 5, 1998.

8. Brandstätter, W., Redl, C. : A LBGK Method for Buoyant Turbulent Combustion Processes. In preparation (2003).

9. Xue, H., Ho, J. C., Cheng, Y. M. : Comparison of different combustion models in enclosure fire simulation. Fire Safety Journal 36 (2001), 37-54.

10. Centre d'Etudes des Tunnels : Les Etudes Specifiques des Dangers pour les Tunnels du Resau Routier. Report Ministere de l'Equipement, des Transports et du Logement (2001), 98 pp.

11. Karki, K. C., Patankar, S. V., Rosenbluth, E. M., Levy, S. S. : CFD Model for Jet Fan Ventilation Systems. Proceedings of 10th International Sysposium on Aerodynamics and Ventilation of Vehicle Tunnels, Boston, November 1-3, 2000.

12. Jones, W. W., Forney, G. P., Peacock, R. D., Reneke, P. A. : A Technical Reference for CFAST : An Engineering Tool for Estimating Fire and Smoke Transport. Report NIST TN 1431 (2000).

13. Formaggia, L., Gerbeau, J. F., Nobile, F., Quarteroni, A. : On the Coupling of 3D and 1D Navier-Stokes Equations for Flow Problems in Compilant Vessels. Comp. Meth. Appl. Mech. Eng. 1991 (2001), 561-582.

14. http://www.vircinity.com

15. http://www.pdc.kth.se

16. Pucher, K. : Konzepte der Tunnel-Brandrauchentlüftung. Längs-, Quer- oder Vollquerlüftung? Proceedings Austroschutz 99, Österreich, 1999.

17. Brandstätter, W., Mawa-Isaac, E., Redl, C. : CFD Simulations of Fire Hazards in the Mont Blanc and Gleinalm Tunnel. VIRTUALFIRES Project Report 5.1, (2002). 